Amplified Salience of Somatic Information in Persistent Depression

\author{
Anna Bevan ${ }^{1,2}$, Caitlin Hitchcock ${ }^{1}$, Daniel J Mitchell ${ }^{1}$, and Tim Dalgleish ${ }^{1,2}$ \\ ${ }^{1}$ Medical Research Council Cognition and Brain Sciences Unit, University of Cambridge \\ ${ }^{2}$ Cambridgeshire and Peterborough NHS Foundation Trust
}

Corresponding Author: Anna Bevan (anna.bevan@mrc-cbu.cam.ac.uk)

This work was funded by the UK Medical Research Council (Grant Reference: SUAG/043 G101400) and supported by the National Institute for Health Research Cambridge Biomedical Research Centre. 


\begin{abstract}
Disrupted bodily awareness may reflect mechanisms which drive both symptomatology and disorder maintenance in clinical depression. We investigated attentional capture by somatic signals in clinically depressed individuals and healthy controls as one aspect of bodily awareness. Attentional performance in a cross-modal covert orienting task was globally disrupted when depressed participants attempted to ignore uninformative somatic cues, in a largely modality-specific manner. In a subsequent study employing a similar paradigm, attention was disproportionately captured in depressed participants by informative somatic cues, in a similarly modality-specific way. Taken together, the results suggest that the salience of somatic signals is amplified in clinical depression, and that attempts to ignore them may have a disruptive effect on attentional processing more generally in this population.
\end{abstract}

Keywords: depression, attention, bodily awareness, salience, covert orienting 


\section{Amplified Salience of Somatic Information in Persistent Depression}

\section{Introduction}

The mind-body problem is at the heart of the enigma of clinical depression. Depression is characterized by physiological changes in crucial allostatic systems including the stress system and the immune system (e.g., Dantzer, O'Connor, Freund, Johnson \& Kelley, 2008; Pariante \& Lightman, 2008; Wohleb, Franklin, Iwata, \& Duman, 2016) but is nevertheless as responsive to psychological as it is to pharmacological intervention (e.g., DeRubeis, Siegle, \& Hollon, 2008; Dimidjian et al., 2006; Kuyken et al., 2015). Moreover, effective psychological interventions succeed in ameliorating the somatic symptomatology of depression despite explicitly focusing upon cognitive, behavioural or interpersonal, rather than somatic, targets for change (National Institute for Health and Care Excellence, 2009). Notwithstanding its peripheral role in psychotherapeutic formulations of depression, it is clear that altered somatic experience is a hallmark of depression at a subjective as much as at a neurobiological level: individuals across cultures and treatment settings commonly describe multiple unexplained somatic symptoms, which in the majority of cases present as the primary reason for treatment-seeking (Kirmayer, Robbins, Dworkind, \& Yaffe, 1993; Simon, VonKorff, Piccinelli, Fullerton, \& Ormel, 1999; Vaccarino, Sills, Evans, \& Kalali, 2008). Somatic symptoms are also associated with depression maintenance (Hung, Liu, Wang, Juang, \& Yang, 2010) and treatment resistance (Papakostas et al., 2003) suggesting that a better understanding of the processes involved may be highly relevant for treatment development.

Altered somatic experience in depression takes a variety of forms. Sensations of exhaustion, heaviness, weakness, illness, tension, aches and pains and a general loss of vitality are commonly reported and may be intrusive and disabling (e.g., Ratcliffe, 2014; Vaccarino et al., 2008). Appetitive sensation (hunger, desire, enjoyment, curiosity, interest and motivation) may, on the other hand, be attenuated or lost. Depressive presentations can in fact be characterized by emotional numbing regardless of emotion valence (e.g., Bylsma, Morris \& Rottenberg, 2008) with more severe 
manifestations of attenuation of emotional or somatic experience apparent in the overlap between depression, alexithymia (Leweke, Leichsenring, Kruse \& Hermes, 2012) and depersonalization (Hunter, Sierra, \& David, 2004). To complicate the picture further, there is evidence that positive relationships may exist in depressed populations between amplified and attenuated forms of somatic sensation, and these are in need of explication (e.g., Sayar, Kirmayer \& Taillefer, 2003).

It has been proposed (Barrett, Quigley, \& Hamilton, 2016; Paulus \& Stein, 2010; Seth \& Friston, 2016) that the emotional and somatic consequences of clinical depression are the result of disrupted interoceptive processing arising in the course of depressive illness. Interoception - the moment-by-moment neural representation of internal bodily signals - is emerging as an important topic of research (e.g., Craig, 2009; Khalsa et al., 2018), and has been hypothesized to provide the basis for allostatic behaviour (Barrett et al., 2016), emotion experience (James, 1894; Schachter \& Singer, 1962), effective decision-making (Damasio, 2006; Dunn et al., 2010), social cognition (Adolfi et al., 2017), conscious awareness (Craig, 2009), and the sense of self (Seth, 2013); all domains which are arguably disturbed in major depression.

In support of this theory, a number of studies (Dunn, Dalgleish, Ogilvie, \& Lawrence, 2007; Furman, Waugh, Bhattacharjee, Thompson, \& Gotlib, 2013; Terhaar, Viola, Bar, \& Debener, 2012) have found evidence for reduced interoceptive accuracy in some depressed populations, as measured by the heartbeat perception test (Schandry, 1981) which quantifies individual differences in terms of the difference between actual and counted heart beats over brief periods of time. High accuracy in this task is assumed to reflect a trait-like tendency to high interoceptive awareness (Ainley, Apps, Fotopoulou, \& Tsakiris, 2016; Mussgay, Klinkenberg, Rüddel, \& D'Agata, 1999) and in healthy participants has been related to theoretically relevant constructs such as emotion regulation (Fustos, Gramann, Herbert, \& Pollatos, 2013) and decision-making acumen (Dunn et al., 2010). These findings are encouraging, but the insights that they offer into the specific mechanisms driving such outcomes are limited because endogenously-occurring stimuli such as heartbeats cannot be (non- 
invasively) experimentally manipulated with precision, and because they are difficult to match with control stimuli to account for supramodal attentional and executive processes.

Signal detectability in general depends on the baseline intensity of the signal relative to system noise, and also on the precision with which it is attended. Interoceptive awareness, therefore, depends both on the baseline intensity of interoceptive signals arising on a moment-by moment basis, and on the degree to which an individual is attending to those signals. Deficits in interoceptive accuracy could arise due to reduced signal/noise ratios (whether due to an attenuated signal or to a noisy context) rendering interoceptive signals hard to differentiate, or because of difficulties in directing and maintaining attention to interoceptive signals to amplify their precision. A further relevant distinction is between endogenous (top-down) and exogenous (stimulus-driven) forms of attention, which evidence suggests are distinct processes relying on partially segregated brain networks (Chica, Bartolomeo, \& Lupiáñez, 2013; Corbetta \& Shulman, 2002). In the heartbeat perception test, top-down attention to interoceptive sensation is recruited via experimenter instruction. But day-to-day interoceptive awareness must surely depend to a much greater extent upon the stimulus-driven likelihood of reorienting attention to interoceptive signals which have only an incidental relation to ongoing task-related priorities.

To understand whether and how bodily awareness is altered in depression, three mechanisms must therefore be delineated: baseline bodily signal intensity or discriminability; the ability to voluntarily direct top-down attention to bodily signals; and the extent of stimulus-driven attentional capture by such signals in the absence of voluntary attention. Given the pace at which the field of interoceptive research is moving and the methodological innovations which are being developed (Khalsa et al., 2018), direct means of studying such distinct mechanisms in interoception may soon be available. In the meantime, we have addressed these questions using an analogue approach employing exteroceptive somatic stimuli. The extent to which exteroceptive somatic processing overlaps with interoceptive processing, and the implications of any such overlap for the understanding of key processes in depression, remain open questions. However, recent advances in 
our understanding of the relationship between interoceptive and exteroceptive somatic processing at a cortical level (discussed below) support the suggestion that cutaneous somatic stimuli can provide a relevant analogue.

Interoceptive and exteroceptive somatic signals are conveyed from peripheral receptors to the cortex via anatomically distinct afferent pathways. Exteroceptive signals include fine and discriminative cutaneous touch, vibration and proprioception, and are conveyed from receptors in the skin and joints via the dorsal column medial lemniscus pathway, which terminates in primary and secondary somatosensory cortices. Interoceptive signals include cutaneous sensations of heat, pain, itch, and crude touch in addition to muscular and visceral sensations, and are conveyed via a phylogenetically recent lamina I spinothalamic pathway terminating in posterior insular cortex (Craig, 2002). Despite the lack of a direct connection between the medial lemniscal pathway and insular cortex, there is mounting evidence, both structural (Cerliani et al., 2012; Cloutman, Binney, Drakesmith, Parker, \& Lambon Ralph, 2012; Ghaziri et al., 2015; Jakab, Molnar, Bogner, Beres, \& Berenyi, 2012) and functional (Cauda et al., 2011; Deen, Pitskel, \& Pelphrey, 2011; Wiech, Jbabdi, Lin, Andersson, \& Tracey, 2014) suggesting that exteroceptive somatic signals are also forwarded from somatosensory cortices to the posterior insula via a ventral somatosensory pathway (Dijkerman \& de Haan, 2007; Preusser et al., 2015). Craig (2010) reviews evidence that the posterior insula may support an array of modality-specific, topographically organized primary interoceptive representations; while evidence from functional imaging studies demonstrates that exteroceptive forms of somatic stimulation including touch (Davis, Kwan, Crawley, \& Mikulis, 1998; Hu et al., 2015; Mazzola, Faillenot, Barral, Mauguiere, \& Peyron, 2012) and vibration (Burton, Videen, \& Raichle, 1993; Coghill et al., 1994; Francis et al., 2000; Golaszewski et al., 2006; Siedentopf et al., 2008) are similarly associated with posterior insula activity. Studies directly comparing insula activity in response to exteroceptive and interoceptive stimulation have identified areas of overlap (Eickhoff et al., 2006; Ostrowsky et al., 2002; zu Eulenburg, Baumgartner, Treede, \& Dieterich, 2013), and such findings, in addition to neuropsychological evidence (Ibanez, Gleichgerrcht, \& Manes, 2010)), have 
led commentators to propose that mid-posterior insular cortex mediates the integration of exteroceptive, interoceptive and vestibular somatic information to form a multimodal body representation (Craig, 2010; Eickhoff et al., 2006; zu Eulenburg et al., 2013). Craig (2010) further suggests that this integrated body representation is subsequently re-represented in anterior insula to form a consciously accessible subjective feeling state. The likelihood, therefore, that exteroceptive and interoceptive somatic representations are integrated at a cortical level at an early stage relative to conscious bodily awareness provides a rationale for the use of exteroceptive somatic stimuli as an alternative to visceral stimuli in the study of somatic awareness in depression.

On this basis, we have employed exteroceptive somatic stimuli in a series of studies, including the present research, with two broad aims: first, to demonstrate the utility of manipulating exteroceptive somatic stimuli to understanding bodily processing; and second, to attempt to dissociate baseline signal intensity, top-down attention to sensory signals, and stimulus-driven attentional capture, in clinically depressed versus healthy participants. In all of these studies we have included comparable control conditions employing auditory stimuli. In our initial studies (Bevan, 2020) we found no support for a shift in baseline signal detectability in clinical depression with respect to either somatic or auditory targets, and, while reliable disturbances in top-down attention to sensory targets were present in our depressed samples, these were not modalityspecific. In sum, we have no evidence for an association between clinical depression and either reduced signal/noise ratios in somatic target detection, or specific disruption in voluntary attention towards such targets. The current studies build on this work by investigating the extent of stimulusdriven attentional capture by somatic signals. In particular, we seek to address three specific questions. First, is persistent depression associated with a shift in the strength of stimulus-driven attentional capture by somatic signals? Second, is any such shift genuinely modality-specific, i.e., non-attributable to the known effects of major depression on supramodal executive and attentional capacities? And finally, what are the implications of any such shift in terms of the salience of somatic 
experience in persistent depression? Specifically, are somatic signals amplified or attenuated through their interactions with attention in depressed relative to healthy individuals?

We investigated these questions using a well-established attention task - the covert orienting paradigm (Posner, 1980; Spence, 2010). In this task, targets are presented either to the left or right of fixation while preceding cues can be either spatially valid (occurring on the same side of space) or invalid (occurring on the opposite side of space) with respect to the target. The reaction time difference between responses to targets where cues are valid and responses where cues are invalid provides an index of attentional capture by those cues, sometimes known as the 'validity effect'. If non-predictive somatic stimuli are employed as cues, this task can therefore yield a measure of the extent of stimulus-driven attentional capture by task-irrelevant somatic information, potentially indexing a key trait or tendency underlying day-to-day bodily awareness. An otherwise identical control condition employs auditory cues to allow us to examine the dissociability of modality-specific from supramodal effects, and thus to account for the influence of general attentional and executive control capacities on task performance.

Study 1 compared the attentional performance of persistently depressed participants with healthy, never-depressed controls on this task. We predicted that if depressed individuals are habituated to ignoring or blocking out somatic signals (attenuated bodily awareness) they would demonstrate a reduced validity effect for somatic cues relative to auditory ones; if, on the other hand, they are sensitized to somatic signals (amplified bodily awareness), they would demonstrate an increased validity effect.

\section{Study 1}

\section{Method}

\section{Power Analysis}

An a priori power analysis was conducted using G*Power 3.1. (Faul, Erdfelder, Lang, \& Buchner, 2007) based on an effect size estimate derived from a visuospatial covert orienting study 
which compared validity effects between depressed and healthy participants (Pardo et al., 2006). The effect size of the group comparison of validity effects in this study was large $\left(\eta_{p}^{2}=0.38\right)$. With $\alpha$ $=.05$ the minimum total sample size to achieve power of 0.95 for a two-tailed test is $\mathrm{N}=24$, or 12 participants per group. We supplemented this by a third and sought to recruit 16 participants per group.

\section{Participants}

Participants were 16 individuals currently experiencing a depressive episode of at least moderate severity and additionally meeting diagnostic criteria for Chronic/Recurrent Major Depressive Disorder (MDD). Sixteen never-depressed healthy controls were matched to the depressed group on age, sex, handedness and years of education. Participants were recruited from an established panel of potential research volunteers. MDD and the presence of a current Major Depressive Episode (MDE) were diagnosed via clinical screening assessments including a Structured Clinical Interview for DSM-IV Axis I disorders (SCID-I: First, Spitzer, Gibbon, \& Williams, 2002) administered by a trained research assistant, and a second SCID-I mood module conducted by a doctoral-level clinical psychologist on the day of testing. Depression and anxiety severity were estimated using the Beck Depression Inventory (BDI-II: Beck, Steer, Ball, \& Ranieri, 1996) and the Beck Anxiety Inventory (BAI: Beck, Epstein, Brown, \& Steer, 1988). All depressed participants scored 20 or above on the BDI-II on the day of testing. Exclusion criteria included alcohol or substance dependence or a history of psychosis, bipolar disorder, traumatic brain injury, intellectual disability or dementia. Exclusion criteria were similar for the healthy control group, with the additional specification that controls had no significant history of anxiety or depression and scored within the 'minimal' range on both self-report measures at the time of testing.

Handedness was assessed using the Edinburgh Handedness Inventory (Oldfield, 1971), and an estimate of years of education was established via a brief conversation with the participant. 
All participants provided written informed consent before taking part, and received compensation of $£ 6$ per hour for their participation. The studies were conducted in accordance with ethical approval from the Cambridge Psychology Research Ethics Committee (PRE.2013.72).

\section{Attention Task}

This task consisted of a cross-modal covert orienting task (Posner, 1980; Spence, 2010) employing visual stimuli as targets and somatosensory and auditory stimuli as uninformative cues. Visual targets consisted of 400 ms illuminations of one of two $3 \mathrm{~mm}$ white light emitting diodes (LEDs) positioned to the left and right peripheries of the participant's visual field (see Figure 1 for the layout of the experimental materials). Somatic cues consisted of $400 \mathrm{~ms}$ bursts of $210 \mathrm{~Hz}$ vibration, delivered by one of two $10 \mathrm{~mm}$ vibrating tactors (310-131, Precision Microdrives) affixed to the participant's left and right ventral forearms using surgical tape. Auditory cues consisted of $400 \mathrm{~ms}$ $440 \mathrm{~Hz}$ tones (60dB LAeq) delivered via one of two $50 \mathrm{~mm}$ mylar speakers. LEDs, tactors and speakers were all connected via the parallel port data register to a desktop PC running MATLAB (The Mathworks, 2009a), and were controlled via a program written using Psychtoolbox (Kleiner et al., 2007) to ensure precise stimulus onset and offset latencies.

\section{Figure 1}

Experimental Materials for Studies 1 and 2

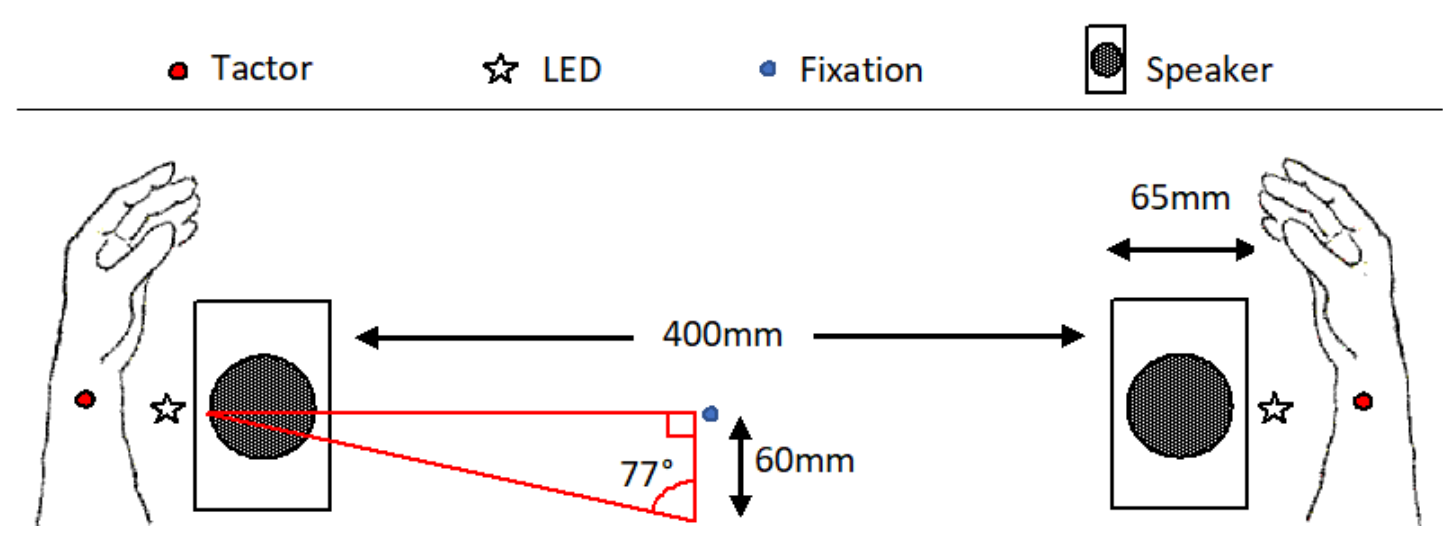


Note. Tactors are affixed to the participant's left and right ventral forearms using surgical tape. The participant's wrists rest against padding fixed to the surface of the table and the outer sides of the speakers. LEDs are embedded in the padding, clearly visible and close to the ipsilateral speaker and tactor.

Trials began with cue onset followed by target onset at one of five possible stimulus-onsetasynchronies (SOAs: $50 \mathrm{~ms}, 100 \mathrm{~ms}, 200 \mathrm{~ms}, 300 \mathrm{~ms}$ or $500 \mathrm{~ms}$ ). A range of SOAs were employed to identify any depression-related timing artifacts that might otherwise be interpreted as absolute group differences. Uncued trials began with target onset. Following target offset, the program paused until a response was made, triggering the start of the next trial. Inter-trial intervals were randomly varied between 1000 and 1500 ms to maintain alertness throughout the trial and prevent participants from predicting target onset. Participant responses were made using foot pedals for the left and right feet, connected to the PC via the parallel port.

\section{Procedure}

Participants were seated at a table, with forearms resting on small pads affixed to the table and eyes resting on a central fixation dot (see Figure 1). Foot pedals were adjusted to a comfortable position under the table and room lights were dimmed during the task. Participants were instructed to maintain fixation on the dot while responding as quickly and as accurately as possible to peripheral visual targets, and to ignore all other stimuli. Two brief practice blocks were completed in order to check participants' understanding of the task and enable them to become familiar with the equipment, particularly the foot pedals. The experimenter observed the participant throughout the task to ensure that central fixation was maintained.

In each trial, a visual target was presented to either the right or left side of fixation, and participants indicated the location of the target as quickly and as accurately as possible using a 
spatially congruent foot pedal response. A spatially valid $(50 \%)$ or invalid (50\%) cue was presented immediately before the target in either the auditory or the somatic modality, depending on block. There were thus two levels of stimulus validity (valid / invalid), two levels of target laterality (left / right), and two levels of cue modality (auditory/somatic). Within each block, five levels of stimulus onset asynchrony (SOA) between cue and target were employed as described above. Eight trials were presented for each combination of SOA, validity and laterality, and there were additionally eight uncued trials for each laterality, totalling 176 trials per block. Trial types (validity, laterality) were presented within blocks in a randomized order, and in total participants completed 4 blocks ( 2 somatic, 2 auditory) in counterbalanced order. Reaction times and error data were collected by the program. During somatic blocks, continuous white noise was delivered via the peripheral speakers to mask any sound associated with tactor vibration.

\section{Results}

\section{Participant Characteristics}

All depressed participants were diagnosed with chronic, recurrent and/or treatmentresistant forms of depressive illness. Mean age of onset was 21.73 years old (SD 11.25) and the mean number of years since first diagnosis was 19.60 (SD 15.89). Six participants (37.5\%) reported that their first onset of depression was in childhood. Six individuals (37.5\%) met criteria for at least one comorbid Axis 1 disorder at the time of testing ( 5 met criteria for 1 or 2 anxiety disorders, including Generalised Anxiety Disorder, Obsessive-Compulsive Disorder and Posttraumatic Stress Disorder, and 1 met criteria for an eating disorder). Six participants (37.5\%) were currently prescribed antidepressant medication. No control participants were prescribed any form of psychotropic medication.

A series of $t$-tests demonstrated that the depressed and healthy control groups were comparable with respect to the characteristics of age, years of education and handedness (see Table 
1). As expected, depressed participants differed significantly from healthy controls with respect to self-reported symptoms of depression and anxiety.

Table 1

Study 1 Demographic Characteristics and Mood Ratings

\begin{tabular}{lcccc}
\hline Characteristic & Depressed group & Control group & & $p$ \\
& & & $t$ & - \\
\hline Sex & 15 women & 15 women & - & 0.94 \\
Age (years) & $42(18.11)$ & $42.5(17.41)$ & -0.08 & 0.41 \\
Handedness (EHI) & $68.14(47.75)$ & $80.06(31.16)$ & -0.84 & 0.64 \\
Education (years) & $15.25(2.86)$ & $15.69(2.41)$ & -0.47 & $<0.001$ \\
Depression (BDI-II) & $33.19(5.06)$ & $5.06(2.70)$ & 20.77 & $<0.001$ \\
Anxiety (BAI) & $22.00(10.00)$ & $3.06(2.64)$ & 7.32 & \\
\hline
\end{tabular}

$\overline{\text { Note. Standard deviations are presented in parentheses. EHI= Edinburgh Handedness Inventory, BDI- }}$ II=Beck Depression Inventory-II; BAI= Beck Anxiety Inventory.

${ }^{a} n=16$ for each group.

\section{Attention Task}

Data Cleaning. Reaction times (RTs) greater than 1500 ms were assumed to reflect equipment failure or inadvertent misuse (typically a failure to fully depress the foot pedal), and trials with these latencies were eliminated from both the RT and error data. Subsequently, responses in which RTs were more than 3 standard deviations away from the mean for each participant (assumed to reflect anticipations and misfires) were eliminated from both RT and error data. Individualized rather than generic cut-offs were employed due to a high level of between-subject variability. In 
total, $2.48 \%$ of responses were disallowed, with no significant effect of Group on the total number of disallowed trials, $t(30)=1.16, p=0.26, d=0.41$. Examination of the remaining data revealed an acceptable error rate of $0.80 \%$. Incorrect responses were eliminated from the RT data. In total, 21,795 observations remained.

No statistically significant interactions between group and SOA were found in initial analyses (all ps > 0.08); therefore, for simplicity, SOA categories are collapsed in the analysis reported here. Uncued trials are not analysed.

Reaction Time Data. The RT data (presented in Table 2) were subjected to a repeatedmeasures ANOVA with the factors of cue Modality, Block number, cue Validity, target Laterality and Group.

Table 2

Study 1 Mean Reaction Time Data by Group and Condition

\begin{tabular}{|c|c|c|c|c|}
\hline \multirow[t]{4}{*}{ Group } & \multicolumn{2}{|c|}{ Valid Cue } & \multicolumn{2}{|c|}{ Invalid Cue } \\
\hline & Left Target & Right Target & Left Target & Right Target \\
\hline & \multicolumn{4}{|c|}{ Auditory Cue } \\
\hline & \multicolumn{4}{|c|}{ Block 1} \\
\hline Depressed & $449.05(76.48)$ & $442.97(77.70)$ & $484.64(86.33)$ & $474.98(73.12)$ \\
\hline \multirow[t]{2}{*}{ Control } & 408.14 (81.01) & 402.40 (84.78) & $435.90(90.13)$ & 431.72 (95.59) \\
\hline & \multicolumn{4}{|c|}{ Block 2} \\
\hline Depressed & $432.88(61.23)$ & 427.15 (70.41) & $471.77(68.37)$ & 469.09 (75.19) \\
\hline Control & $395.85(62.30)$ & 379.65 (53.95) & $421.48(60.45)$ & 407.64 (60.69) \\
\hline
\end{tabular}




\begin{tabular}{lcccc}
\hline Depressed & $465.36(74.63)$ & $452.37(68.78)$ & $505.78(73.57)$ & $487.14(62.95)$ \\
Control & $394.48(73.14)$ & $377.55(72.33)$ & $426.40(79.84)$ & $414.54(79.72)$ \\
\cline { 2 - 5 } & & \multicolumn{3}{c}{ Block 2 } \\
\hline Depressed & $440.46(58.17)$ & $434.27(72.11)$ & $485.43(68.32)$ & $476.13(78.79)$ \\
Control & $384.15(60.52)$ & $371.86(58.97)$ & $409.99(65.81)$ & $400.30(64.44)$ \\
\hline
\end{tabular}

Note. Standard deviations are presented in parentheses.

Within-Participants Effects. The ANOVA revealed that there was no significant main effect of Modality, $F<1$, indicating that on average participants responded with comparable speed during somatic and auditory blocks. A main effect of Block, $F(1,30)=8.78, p=0.006, \eta_{p}{ }^{2}=0.23$, indicated that responses were speeded during later blocks relative to earlier ones, probably reflecting a practice effect. As expected, there was a large (Cohen, 1988) main effect of Validity, $F(1,30)=97.74$, $p<0.001, \eta_{p}^{2}=0.77$, demonstrating that invalid cues were associated with slowed responses relative to valid cues. There was also an effect of Laterality, $F(1,30)=6.05, p=0.02, \eta_{p}{ }^{2}=0.17$, indicating that left-sided responses were somewhat slowed relative to right-sided responses. There were no other statistically significant within-participants effects (all $F_{s}<1.87$; all $p s>0.1$ ).

Group Effects. There was a significant main effect of Group, indicating that depressed participants responded on average more slowly than healthy controls, $F(1,30)=6.36, p=0.017, \eta_{p}^{2}$ $=0.18$. Importantly, an interaction between Modality and Group, $F(1,30)=9.64, p=0.004, \eta_{p}{ }^{2}=$ 0.24 , indicated that the slowing in the depressed participants was especially strong in the somatic modality, $F(1,30)=9.89, p=0.004, \eta_{p}{ }^{2}=0.25$, and did not reach statistical significance in the auditory modality, $F(1,30)=3.55, p=0.069, \eta_{p}{ }^{2}=0.11$ (see Figure 2 , upper left panel). A 3-way interaction between Block, Validity and Group, $F(1,30)=4.98, p=0.033, \eta_{p}^{2}=0.14$, reflected increased validity effects in the depressed group relative to healthy controls in later blocks, $F(1,30)=$ 5.05, $p=0.032, \eta_{p}^{2}=0.14$, but not in earlier ones, $F(1,30)=0.28, p=0.60, \eta_{p}^{2}=0.009$ (Figure 2, 
lower right panel). There were no other statistically significant interactions between group and any other effect (all $F_{s}<1.43$, all $p s>0.1$ ).

Figure 2

\section{Study 1 Outcomes}

Group by Modality Interaction RTs

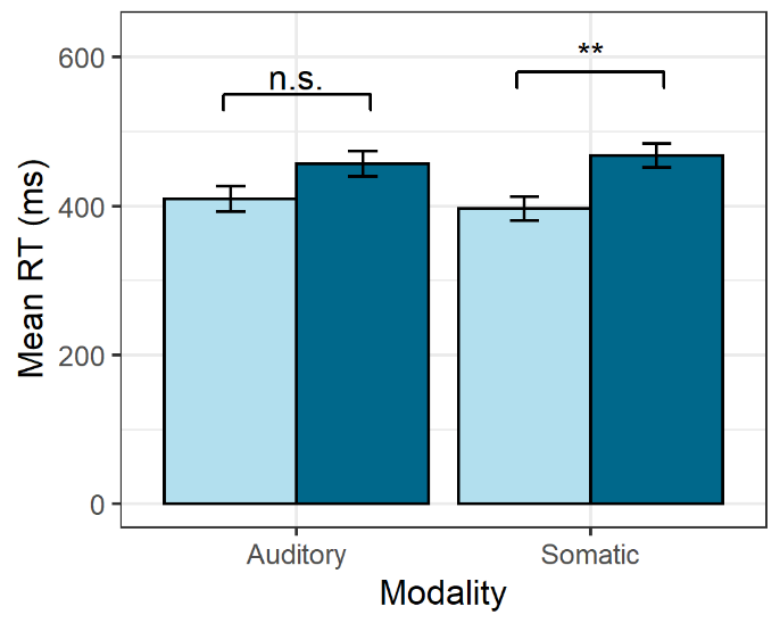

Group by Modality Interaction Intra-Individual Variability

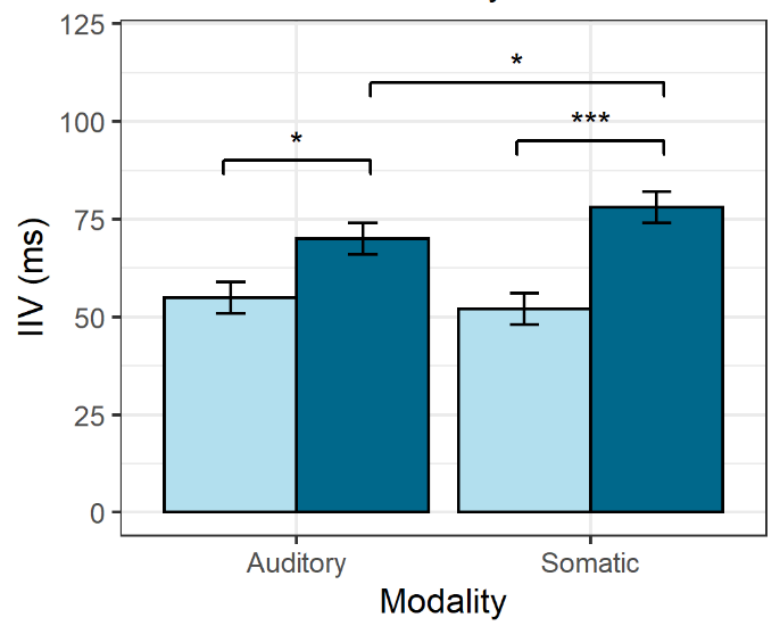

Group by Modality Interaction Errors

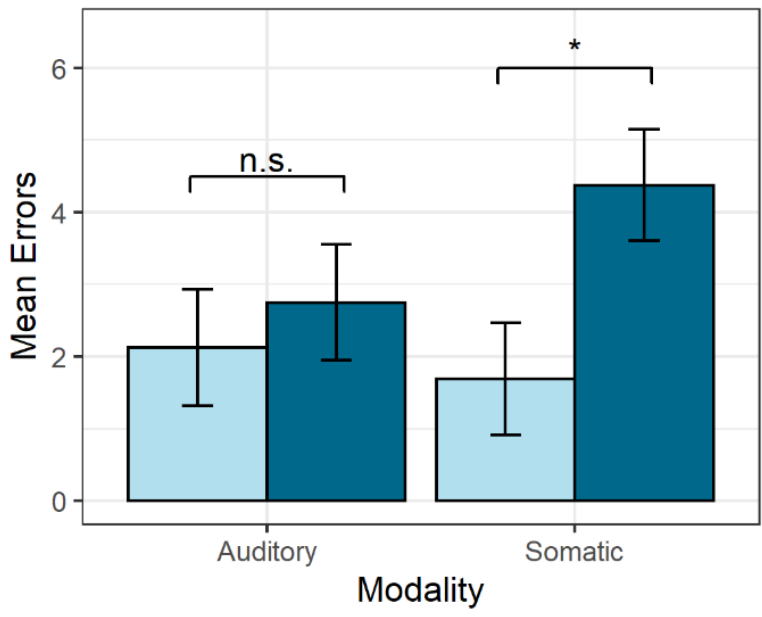

Group by Block Interaction Validity Effect

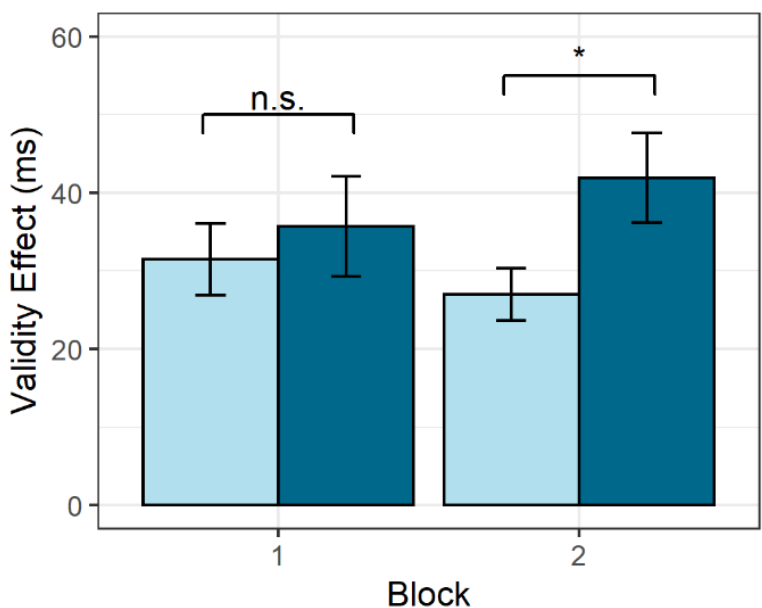

Depressed

Note. Error bars represent one standard error of the mean. Asterisks represent $p$-values less than $0.05(*), 0.01\left({ }^{* *}\right)$ and $0.001\left({ }^{* *}\right)$. The upper left panel shows an interaction between Group and Modality in overall reaction time (RT), indicating relatively slowed responses among depressed 
participants following somatic cues. The upper right and lower left panels show the same effect respectively in error rates (depressed participants are more prone to errors following a somatic cue) and in intra-individual variability (depressed participants are more variable in their responses following a somatic cue). The lower right panel shows the interaction between Group, Validity and Block, indicating that depressed participants displayed increased validity effects relative to controls during later blocks of the task.

RT Data Summary. In summary, for the RT data an interaction between group and cue modality indicated a specific disadvantage for depressed individuals when cues were somatic, regardless of cue validity. Both groups demonstrated speeded responses in later blocks relative to earlier ones, suggestive of a practice effect; and both groups showed a modest disadvantage for leftsided responses, possibly muscular, given that a predominantly right-handed sample is likely to be predominantly right-footed also (Augustyn \& Peters, 1986). The expected effect of cue validity was strongly present in both groups and exacerbated in depressed participants relative to healthy controls in later blocks, possibly indicating a group-specific change in supramodal attentional control over time. There was no evidence, however, of differential validity effects for somatic versus auditory cues across groups.

Error data. Given the significant Modality by Group interaction in the RT data, errors were examined to rule out the possibility of a speed-accuracy trade-off. Error data were collapsed together into modality categories and a Modality by Group ANOVA was conducted on the resulting scores. The data are shown in the upper right panel of Figure 2.

There was no significant main effect of Modality, $F(1,30)=1.58, p=0.22, \eta_{p}{ }^{2}=0.05$, and no significant main effect of Group, $F(1,30)=2.67, p=0.11, \eta_{p}{ }^{2}=0.08$. There was, however, a significant interaction between Modality and Group, $F(1,30)=4.78, p=0.037, \eta_{p}{ }^{2}=0.14$, in the same direction as that found in the RT data; i.e., the depressed group made more errors following 
somatic cues (see Figure 2, upper right panel). Independent samples $t$-tests confirmed that the depressed group made significantly more errors in the somatic condition than did controls, corrected $t(17.89)=2.46, p=0.024, d=0.87$, with no evidence of any group difference in error rates in the auditory condition, $t(30)=0.55, p=0.59, d=0.19$.

Intra-individual Variability (IIV). The pattern of slowed reaction times and increased error rates in the depressed group specifically in response to somatic cues suggests a reduction in attentional efficiency under these circumstances. This hypothesis was investigated further by developing an index of intra-individual variability (IIV) in RT scores. IIV is a phenomenon which is thought to reflect attentional control, such that increased variability is associated with reduced control or efficiency (e.g., Bellgrove, Hester, \& Garavan, 2004; Weissman, Roberts, Visscher, \& Woldorff, 2006).

Modality-specific IIV scores were determined for each participant by calculating the standard deviation of RTs within each trial category and averaging across all categories within each modality. These data are shown in the lower left panel of Figure 2. A Modality by Group ANOVA was then conducted on the IIV scores.

The ANOVA found no main effect of Modality, $F(1,30)=1.48, p=0.23, \eta_{p}{ }^{2}=0.05$. There was, however, a large main effect of Group, $F(1,30)=15.33, p<0.001, \eta_{p}{ }^{2}=0.34$, indicating increased IIV among depressed participants relative to healthy controls. There was also a significant interaction between Modality and Group, $F(1,30)=5.60, p=0.025, \eta_{p}{ }^{2}=0.16$, indicating a disproportionate increase in IIV during somatic blocks in the depressed group (see Figure 2, lower left panel).

Independent samples $t$-tests confirmed that IIV was increased in depressed participants relative to healthy controls in the auditory condition, $t(30)=2.49, p=0.019, d=0.87$, but that the effect was more marked in the somatic condition, $t(30)=4.98, p<0.001, d=1.71$. Furthermore, depressed individuals were significantly more variable in their responses in the somatic condition than they were in the auditory condition, $t(15)=2.16, p=0.048, d=0.44$, while the variability 
displayed by healthy controls did not significantly differ across modalities, $t(15)=1.03, p=0.32, d=$ 0.23 .

Medication-Free Sensitivity Analyses. To rule out the potentially confounding factor of medication status, all analyses were repeated excluding $(n=6)$ depressed individuals who were prescribed psychotropic medication at the time of the study. The results are detailed in the Supplemental Materials. All key group effects remained statistically significant, with the exception of the error analysis, in which statistical significance did not survive a correction for unequal variances, due to the reduced power, but effect sizes remained large. In summary, there is no evidence to suggest that group effects in this study are attributable to psychotropic medication status.

Correlation analysis. An additional analysis was undertaken to rule out the possibility that the observed effects of modality on performance in the depressed group were accounted for by anxiety symptomatology rather than by depression per se. The somatic IIV score was used as the dependent variable because this outcome represented the most marked difference between groups. Partial correlations demonstrated that the relationship between somatic IIV and BDI-II score, controlling for BAI score, remained significant, $r(29)=0.55, p=0.001$, while the relationship between somatic IIV and BAI score, controlling for BDI-II score, was non-significant, $r(29)=-0.10, p=$ 0.59 .

\section{Discussion}

The results of Study 1 demonstrate a marked and largely specific effect of uninformative somatic cues on general attentional performance in the depressed group relative to healthy controls. Spatially specific attentional capture in the depressed group was unaffected by cue modality: instead, decrements in performance indexed by global markers of attentional control, including longer reaction times, higher error rates and increased intra-individual variability, were observed in depressed participants within the somatic condition regardless of validity. This pattern of responses, particularly RT variability, has been interpreted in relevant literatures as diagnostic of a 
breakdown in the efficiency of attention regulation (e.g., Bellgrove et al., 2004; Kelly, Uddin, Biswal, Castellanos, \& Milham, 2008; Sonuga-Barke \& Castellanos, 2007). Reduced attentional efficiency in depression is not, in itself, a novel finding; indeed, concentration difficulties are a considered to be a core symptom of the illness (DSM-V: American Psychiatric Association, 2013), but to our knowledge this is the first report demonstrating that such problems are accentuated in the somatic modality.

In the absence of a significant interaction between Group, cue Modality and Validity, the question of whether depression is associated with an increase or a decrease in the salience of somatic signals cannot be directly answered. Sensitization to somatic signals might draw attention away from the visual targets and entail greater effort to ignore them; conversely, attenuation or habitual ignoring might weaken their alerting effect, reducing anticipation of and preparation for the impending target, while increasing the risk of mind-wandering. Either situation could account for the pattern of results seen here. In addition, a possible alternative explanation for these findings is that white noise (played continuously during somatic blocks only) could have been particularly distracting for depressed individuals and responsible for their performance decrements observed during these blocks. A second experiment was therefore conducted to clarify these issues.

\section{Study 2}

In Study 2, the direction of any interaction between cue modality and salience-driven attentional capture was examined in detail by manipulating cue informativeness. Informative or predictive cues (for example, cues that are valid $75 \%$ of the time) are advantageous to attend and therefore less likely to be ignored than uninformative cues such as those employed in Study 1. In a task employing informative cues, the amplification and attenuation hypotheses generate opposing predictions. The amplification hypothesis suggests that predictive somatic cues will be highly salient and therefore predicts increased validity effects in depressed participants relative to healthy controls. The attenuation hypothesis predicts that depressed individuals will be less able to utilize predictive somatic cues and will therefore demonstrate reduced validity effects relative to healthy 
controls. The results of this experiment should therefore provide clear support for one hypothesis over the other. Additionally, to rule out the alternative white noise explanation for reduced attentional efficiency in depression, white noise was continuously delivered during all blocks in Study 2, regardless of cue modality.

\section{Method}

\section{Participants}

The characteristics and inclusion and exclusion criteria for the two study groups were as for Study 1. Members of the Study 1 depressed sample were invited to participate in Study 2, which took place 6 months later. Of the 16 original participants, 12 responded, including one participant who was in remission from depression, and one who remained depressed but at a mild-moderate level of severity. The remaining 10 responders continued to meet the inclusion criteria and were included in the study. Eight matched controls from Study 1 also returned to participate in Study 2. An additional 6 depressed participants and 8 healthy controls were recruited, using recruitment procedures and inclusion and exclusion criteria identical to those employed in Study 1.

The final sample consisted of 16 individuals in the depressed group meeting diagnostic criteria for chronic/recurrent Major Depressive Disorder (MDD) and experiencing a current Major Depressive Episode, and 16 never-depressed healthy controls matched to the depressed group on age, sex and years of education. Self-report measures were completed as in Study 1.

\section{Attention Task}

Materials, layout, stimulus construction and experimental design parameters other than cue informativeness were identical to those used in Study 1 (see Figure 1). The key manipulation characterizing Study 2 was the use of informative cues, i.e., blocks in which $75 \%$ of cues were valid. During a given block, 12 trials employing valid cues were presented for each level of SOA and laterality, yielding a total of 120 trials. Four trials employed invalid cues for every level of SOA and laterality, yielding a further 40 trials. As in Study 1, eight additional trials per laterality were uncued, 
bringing the total number of trials to 176 per block. Participants were presented with one block in which cues were informative and somatic, and one block in which they were informative and auditory. In addition, one block of uninformative (50\% valid) somatic cues and one block of uninformative auditory cues were presented. Uninformative blocks were identical to those employed in Study 1. The order of block presentation was counterbalanced using a Latin square.

The experimental procedure was similar to that employed during Study 1, with a key difference in the instructions given. In contrast to Study 1 participants, Study 2 participants were informed about cue contingencies at the outset of the experiment and were also told at the beginning of each block whether subsequent cues would be 'helpful' (informative cues) or 'unhelpful' (uninformative cues). No further instructions were given to Study 2 participants regarding how they should respond to the cues.

In contrast to Study 1, white noise was delivered via both external speakers throughout all blocks regardless of cue modality.

\section{Results}

\section{Participant characteristics}

All depressed participants were diagnosed with a current Major Depressive Episode of at least moderate severity, and with Chronic or Recurrent Major Depressive Disorder. Mean age of onset was 28.57 years old $(S D=15.21)$ and the mean number of years since first diagnosis was 23.10 $(S D=16.60)$. Three participants $(18.75 \%)$ reported that their first onset of depression was in childhood. Five individuals (31.25\%) met criteria for at least one comorbid anxiety disorder (including Generalised Anxiety Disorder, Obsessive-Compulsive Disorder, Posttraumatic Stress Disorder and Social Phobia) at the time of testing. Ten participants (62.5\%) were currently prescribed antidepressant medication. One control participant was also prescribed psychotropic medication (amitriptyline for migraine). Independent samples $t$-tests demonstrated no significant group differences in demographic characteristics including age, handedness and years of education. As 
expected there were significant group differences in self-reported depression and anxiety (see Table 3).

Table 3

Study 2 Demographic Characteristics and Mood Ratings

\begin{tabular}{|c|c|c|c|c|}
\hline Characteristic & Depressed group ${ }^{a}$ & Control group $^{a}$ & $t$ & $p$ \\
\hline Sex & 12 women & 12 women & - & - \\
\hline Age (years) & 51.69 (14.79) & $50.31(14.60)$ & 0.27 & 0.79 \\
\hline Handedness (EHI) & $50.76(69.75)$ & $62.34(65.59)$ & -0.48 & 0.63 \\
\hline Education (years) & $15.25(3.34)$ & $16.50(2.28)$ & -1.24 & 0.23 \\
\hline Depression (BDI-II) & $32.63(8.82)$ & $2.06(2.38)$ & 13.39 & $<0.001$ \\
\hline Anxiety (BAI) & $19.96(12.53)$ & $1.44(1.26)$ & 5.88 & $<0.001$ \\
\hline \multicolumn{5}{|c|}{ Note. Standard deviations are presented in parentheses. EHI= Edinburgh Handedness Inventory, BD } \\
\hline II=Beck Depression & -II; BAI= Beck A & tory. & & \\
\hline
\end{tabular}

\section{Attention Task}

Data Cleaning. The data were cleaned according to the criteria employed in Study 1. In total, $2.14 \%$ of responses were assumed to have occurred as a result of anticipations, misfires and equipment failure and disallowed. There was no effect of group on the total number of disallowed trials, $t(30)=0.07, p=0.94, d=0.03$. Examination of the remaining data revealed an acceptable error rate of $0.48 \%$. Incorrect responses were removed from the RT data. In total, 21,940 observations remained. 
As in Study 1, no statistically significant interactions between group and SOA were found in initial analyses ( $p s>0.1$ ); therefore, SOA categories are collapsed in the analysis reported here. Uncued trials were not analysed.

Reaction Time Data. The full RT data are presented in Table 4. These data were subjected to a repeated-measures ANOVA with the factors of cue Informativeness, cue Modality, cue Validity, target Laterality and Group.

Table 4

Study 2 Reaction Time Data by Group and Condition

\begin{tabular}{|c|c|c|c|c|}
\hline \multirow[t]{4}{*}{ Group } & \multicolumn{2}{|c|}{ Valid Cue } & \multicolumn{2}{|c|}{ Invalid Cue } \\
\hline & Left Target & Right Target & Left Target & Right Target \\
\hline & \multicolumn{4}{|c|}{ Auditory Cue } \\
\hline & \multicolumn{4}{|c|}{ Uninformative (50\% valid) } \\
\hline Depressed & $466.02(126.52)$ & 452.59 (116.90) & $495.68(140.46)$ & 491.86 (129.53) \\
\hline \multirow[t]{2}{*}{ Control } & 398.30 (53.59) & 385.45 (43.79) & $431.91(56.28)$ & $410.17(48.24)$ \\
\hline & \multicolumn{4}{|c|}{ Informative (75\% valid) } \\
\hline Depressed & 433.99 (63.81) & 432.59 (60.95) & $479.97(72.35)$ & $491.72(75.70)$ \\
\hline \multirow[t]{3}{*}{ Control } & $398.88(52.68)$ & $381.45(52.65)$ & $435.82(54.18)$ & $426.36(61.85)$ \\
\hline & \multicolumn{4}{|c|}{ Somatic Cue } \\
\hline & \multicolumn{4}{|c|}{ Uninformative (50\% valid) } \\
\hline Depressed & 437.38 (70.29) & 444.95 (76.42) & 485.54 (91.99) & 497.12 (95.36) \\
\hline \multirow[t]{2}{*}{ Control } & 409.63 (70.71) & 394.67 (76.61) & $442.08(68.13)$ & 423.35 (73.67) \\
\hline & \multicolumn{4}{|c|}{ Informative (75\% valid) } \\
\hline Depressed & $451.13(104.76)$ & 455.95 (115.36) & $523.44(116.31)$ & $516.33(141.44)$ \\
\hline
\end{tabular}


Control

$429.08(107.57)$

$413.96(89.94)$

$471.51(105.37)$

$445.78(87.86)$

Note. Standard deviations are presented in parentheses.

Within-Participant Effects. As expected, there was a large main effect of cue Validity, $F(1$, $30)=72.79, p<0.001, \eta_{p}{ }^{2}=0.71$. The ANOVA also revealed a substantial interaction between cue Informativeness and cue Validity, $F(1,30)=18.60, p<0.001, \eta_{p}{ }^{2}=0.38$, indicating that validity effects were larger in blocks where cues were informative. A four-way interaction between Informativeness, Modality, Validity and Laterality, $F(1,30)=5.15, p=0.031, \eta_{p}^{2}=0.15$, indicated that in informative blocks only, validity effects were larger on the left when cues were somatic and larger on the right when cues were auditory. This effect is difficult to interpret on theoretical grounds.

A non-significant trend towards a main effect of cue Informativeness, $F(1,30)=3.26, p=$ $0.081, \eta_{p}^{2}=0.10$, is explained by the Informativeness by Validity interaction described above: i.e., by a systematic disadvantage for the invalid minority, but not the valid majority, of informative cues. There were also non-significant trends towards a main effect of Modality, $F(1,30)=3.50, p=0.071$, $\eta_{p}{ }^{2}=0.10$, an interaction between Modality and Validity, $F(1,30)=3.36, p=0.077, \eta_{p}{ }^{2}=0.10$, and a 3-way interaction between Modality, Validity and Laterality, $F(1,30)=3.57, p=0.069, \eta_{p}^{2}=0.11$. There were no other statistically significant within-participant effects (all $F s<2.9$, all $p s>0.1$ ).

Group effects. Only one statistically significant group effect emerged from the RT analysis: an interaction between Modality, Validity and Group, $F(1,30)=4.65, p=0.039, \eta_{p}^{2}=0.13$. This indicated that depressed individuals demonstrated significantly increased validity effects relative to controls in response to somatic cues, $F(1,30)=4.44, p=0.043, \eta_{p}^{2}=0.13$, but not to auditory cues, $F(1,30)=0.77, p=0.39, \eta_{p}^{2}=0.03$. Furthermore, within the depressed group validity effects were significantly larger in response to somatic cues than to auditory ones, $F(1,15)=5.13, p=0.039, \eta_{p}{ }^{2}=$ 0.26 , whilst validity effects among healthy controls did not differ from one modality to the other, $\left.F(1,15)=0.12, p=0.74, \eta_{p}^{2}=0.008\right)$. See Figure 3 (left panel). 


\section{Figure 3}

\section{Study 2 Outcomes}

Group by Modality Interaction Validity Effect

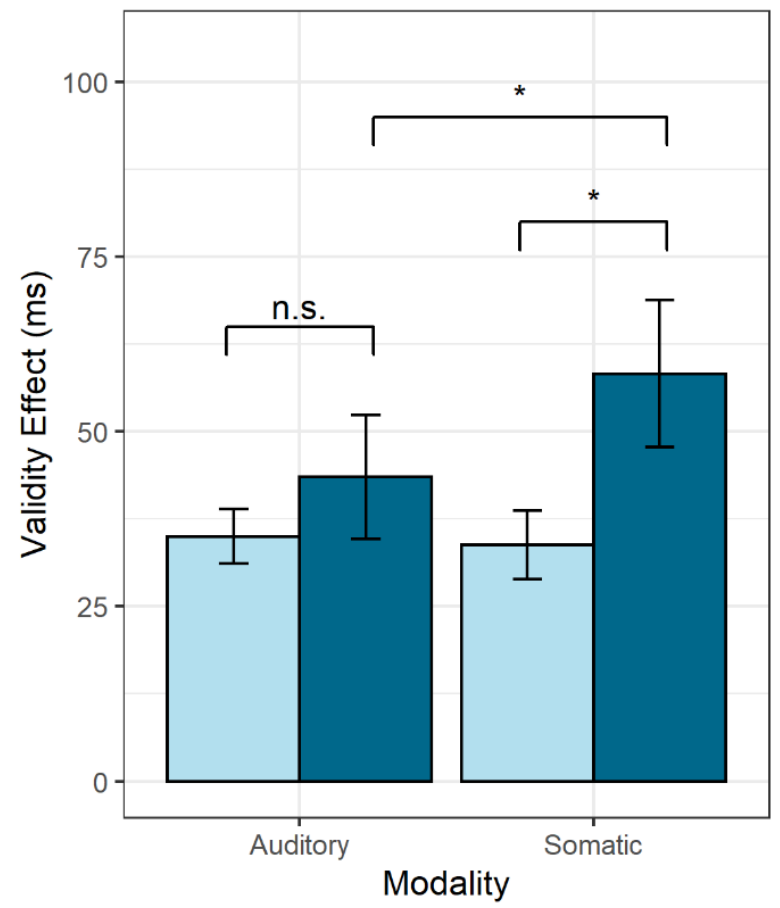

Group by Modality Interaction Error Validity effect

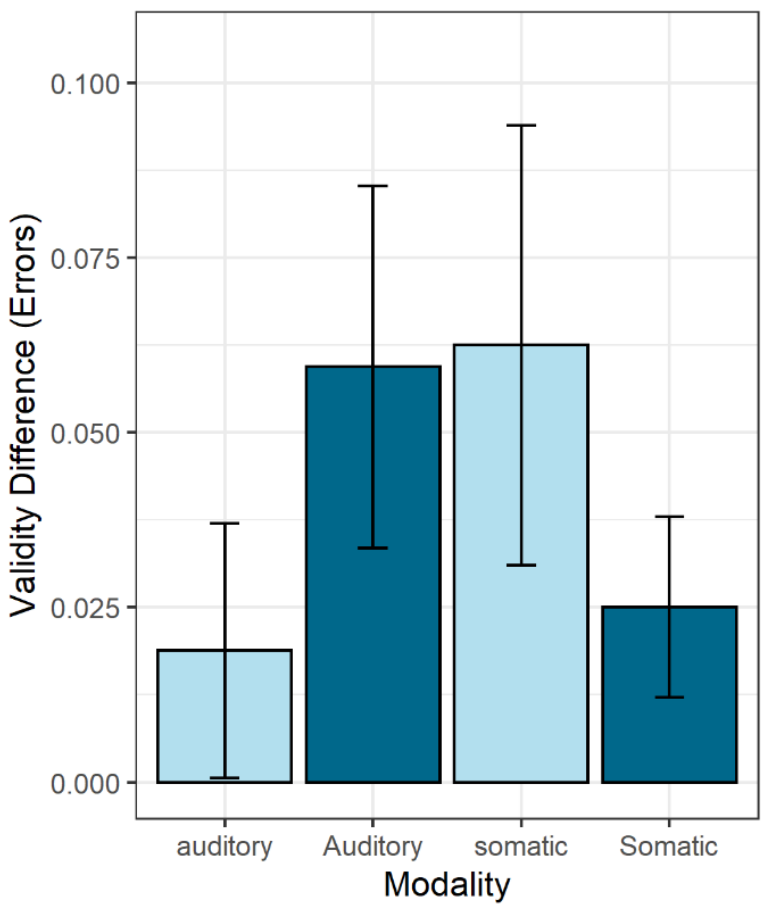

Group $\square$ Control Depressed

Note. Error bars represent one standard error of the mean. Asterisks represent $p$-values less than

0.05. The left panel shows validity effects by Group and Modality, indicating that validity effects following somatic cues are exacerbated in depressed participants relative to healthy controls. Validity effects following somatic cues are significantly larger than those following auditory cues in depressed participants. "Error validity effects" in the right panel were calculated by subtracting errors following valid cues from errors following invalid cues (shown by Group and Modality). This pattern of results is in the opposite direction to the RT outcomes, with depressed participants showing reduced influence of somatic cues on error rates relative to the influence of auditory cues. Control participants show the opposite pattern. 
There was a non-significant trend towards a main effect of Group, $F(1,30)=3.56, p=0.069$, $\eta_{p}^{2}=0.11$, suggesting somewhat slowed responses in the depressed group relative to healthy controls; and a similarly non-significant trend towards an interaction between Group and Laterality, $F(1,30)=3.87, p=0.058, \eta_{p}^{2}=0.11$, suggesting that healthy controls, but not depressed participants, may have had a tendency to respond more slowly to left-sided targets. There were no other significant interactions between group and any other effect (all $F_{s}<2.71$, all $p s>0.1$ ). Of note, in contrast to Study 1, now that informative cues were employed there was no evidence of any significant Group by Modality interaction in RTs as a whole, $F(1,30)=0.59, p=0.45, \eta_{p}^{2}=0.02$.

Error Analysis. Following Study 1, errors were pooled according to cue modality and validity and an ANOVA with the factors of Modality, Validity and Group was performed on the resulting scores. The ANOVA revealed a main effect of Validity, $F(1,30)=7.76, p=0.009, \eta_{p}{ }^{2}=0.21$, indicating that error rates were higher following an invalid cue relative to a valid one. Of interest, there was also an interaction between Group, Modality and Validity, $F(1,30)=7.79, p=0.009, \eta_{p}{ }^{2}=0.21$, indicating that auditory cues had greater influence than somatic cues on error rates among the depressed group, while the opposite pattern was observable in the control group (see Figure 3, right panel). No contrasts between group and condition met statistical significance however ( $p s>0.05)$. There were no other significant effects (all $F s<0.5$, all $p s>0.4$ ).

Intra-Individual Variability (IIV). An ANOVA with the factors of Group, cue Modality, and cue Validity was also conducted on IIV scores, calculated using the method described in Study 1 . The ANOVA revealed a significant main effect of Modality, indicating that somatic cues were associated with greater response variability within the sample as a whole, $F(1,30)=4.54, p=0.041, \eta_{p}{ }^{2}=0.13$. There was also an interaction between Modality and Validity, $F(1,30)=5.15, p=0.031, \eta_{p}{ }^{2}=0.15$, indicating that across the whole sample greater variability was associated with valid auditory cues and with invalid somatic cues. There were no other significant main or interaction effects (all Fs $<$ 2.25, all $p s>0.1$ ). 
Medication-Free Sensitivity Analysis. In order to rule out the potentially confounding factor of medication status on observed group differences, the RT analysis was repeated excluding 10 depressed individuals and 1 member of the control group who were prescribed psychotropic medication at the time of the study. The effect size of the critical group difference (the interaction between Modality, Validity and Group) actually increased, although it no longer reached statistical significance as a result of the markedly reduced power, $\left.F(1,19)=3.78, p=0.067, \eta_{p}{ }^{2}=0.17\right)$. This suggests that medication status is unlikely to account for this finding.

Study Repetition Sensitivity Analysis. Ten depressed participants and eight control participants took part in Study 1 six months prior to participating in Study 2 . In order to estimate the possible impact of study repetition the RT analysis was repeated for two subgroups - those who participated in both studies and those who participated in Study 2 only. The critical group difference (the interaction between Modality, Validity and Group) did not reach statistical significance for either subgroup due to reduced power $(F s<2.4, p s>0.1)$, but effect sizes remained in the medium range for both subgroups: $\eta_{p}{ }^{2}=0.13$ for those who participated in both studies, and $\eta_{p}{ }^{2}=0.11$ for those who participated only in Study 2. There is therefore no evidence that study repetition differentially influenced group performance on this task.

Correlation analysis. As in Study 1, an additional analysis was undertaken to rule out the possibility that the observed group difference in the somatic validity effect was accounted for by anxiety symptomatology rather than by depression per se. Partial correlations demonstrated that the relationship between the somatic validity effect and BDI score, controlling for BAI score, remained statistically significant, $r(29)=0.42, p=0.019$, while the relationship between somatic validity and BAI score, controlling for BDI score, was not, $r(29)=-0.21, p=0.26$.

\section{Discussion}

Study 2, employing informative cues, demonstrated increased validity effects for somatic relative to auditory information in the depressed group, with the implication that attention in this 
group was disproportionately captured by somatic signals. The direction of the effect therefore provides support for the hypothesis that the salience of somatic signals is amplified, rather than attenuated, among depressed participants.

Of interest, the error data also yielded an interaction between group, modality and validity, but in the opposite direction. This "error validity effect" - the result of subtracting error rates following valid cues from error rates following invalid cues - was largest for depressed participants when cues were auditory, with healthy controls showing the opposite pattern. This finding is also consistent with the amplification theory of somatic stimuli in depression, if it is assumed that depressed participants find disproportionately salient somatic cues easier to distinguish from targets than their auditory counterparts. Less distinctive cues may be more frequently mistaken for targets and responded to accordingly, a process which would be expected to yield few errors following valid cues but relatively many following invalid ones. Conversely, a highly salient cue may be readily distinguishable from the target, with the result that error rates are less dependent on cue validity.

In contrast to Study 1, there was no evidence of reduced attentional efficiency in the depressed group overall, despite the fact that white noise was played continuously throughout all blocks. This suggests that white noise was not responsible for the loss of attentional efficiency observed in the depressed group during somatic blocks in Study 1, although as a null result it must be interpreted cautiously. The significant Group by Modality by Validity effect, observed here with constant white noise, also suggests that the modality effect in Study 1 was not dependent on the difference in white noise.

A key remaining question concerns the reason for the different but related outcomes across the two studies. In Study 1 somatic cues increased attentional inefficiency in depressed participants; in Study 2 somatic cues increased attentional capture in the same group. This difference in outcomes could be a result of different instructions: in Study 1, participants were instructed to "ignore" the (non-predictive) cues: increased attentional inefficiency could therefore be a result of 
disproportionate difficulty in doing so, consistent with the hypothesis that somatic cues were perceived as disproportionately salient. In Study 2, no instruction was given with respect to the (predictive on average) cues: increased attentional capture may thus have emerged as a consequence of disproportionate salience uninfluenced by efforts to ignore or redirect attention.

\section{General Discussion}

The two studies described above set out to address three questions: First, to identify whether somatic, as opposed to visceral, paradigms can differentiate between depressed and healthy populations, with the aim of establishing the relevance of such approaches to the study of bodily awareness in depression. Second, to establish whether systematic differences in somatic processing between depressed and healthy groups have a genuinely modality-specific component, beyond a global influence of depression on supramodal executive and attentional capacities. Finally, to investigate the nature of any change in bodily awareness in persistent depression; specifically, whether somatic signals are afforded increased or decreased salience relative to non-somatic auditory signals. These questions will be discussed in turn.

The results of both studies suggest that persistent depression has a measurable impact on aspects of somatic awareness. The interactions between depressed group status and the somatic modality were more marked in both studies than the impact of depression on any other aspect of performance, despite the fact that the task relied on functions such as concentration and speed of processing, well known to be vulnerable to disturbance in depression. These findings provide some evidence for the proposition (Barrett et al., 2016; Paulus \& Stein, 2010) that a disturbance in somatic awareness may be a central attribute of clinical depression. The findings also suggest some possibilities as to the nature of the disturbance. No group differences were previously found in perceptual sensitivity for somatic signals when attention was explicitly directed towards isolated somatic targets in a top-down fashion (Bevan, 2020), but marked group differences emerged here when measuring attentional capture by somatic sensations that preceded visual targets. One 
hypothesis suggested by these findings is that, without necessarily having a direct effect on the fundamental nature or intensity of bodily percepts, persistent depression can nevertheless affect bodily awareness through specific effects on attention towards bodily experience. Altered somatic experience in depression may thus be related to disturbances of attention towards somatic or interoceptive sensation. This proposal has implications for the development of attention-focused psychotherapeutic approaches to somatic symptomatology in depression, and may also be relevant to understanding the mechanisms underlying established attention-focused psychotherapies such as Mindfulness-Based Cognitive Therapy (Kuyken et al., 2015; Segal \& Teasdale, 2018).

The studies provide some evidence, as expected, of reduced attentional capacity at a global level in the depressed group relative to healthy controls. In particular, a group difference in validity effects across modalities was observed during later blocks of Study 1, perhaps indicating a fatigue effect amongst depressed participants. Interestingly, this effect occurred in the context of overall increases in response speed and efficiency in both groups across blocks, which may suggest fatigue or attenuation of a specific attentional mechanism rather than general flagging. Additionally, Study 1 found both increased intra-individual variability in RT and a trend towards RT slowing in the depressed group during auditory blocks as well as somatic blocks, suggesting that to some extent supramodal attentional capacities were implicated. As described above, however, not only were group effects substantially larger during somatic blocks, but within the depressed group itself key indices of attentional capacity (IIV in Study 1 and validity effect in Study 2) were disproportionately increased during somatic blocks relative to auditory ones. Given that within each task the somatic and auditory conditions were largely identical, these findings argue in favour of genuinely modalityspecific effects of somatic processing on attentional performance in depression.

A possible non-specific explanation is that a modality processing differential common to all participants is exacerbated in depression (if, for example, somatic information represents greater attentional load, depressed individuals could be disproportionately affected due to reduced global processing capacity), but this explanation is unlikely since healthy controls showed few effects of cue 
modality in their responses. Taken together, the results of both studies are most parsimoniously explained by the hypothesis that somatic information exerts a particular, modality-specific influence on generic attentional capacities in major depression.

An additional question posed in the current studies relates to the nature and direction of this influence. Existing formulations of disturbed somatic processes in depression posit an attenuation of somatic experience (e.g., Bylsma et al., 2008), although the self-reports of depressed individuals demonstrate that at least some forms of unpleasant somatic experience can be highly salient during depressive episodes (Ratcliffe, 2014). While a disturbance of somatic awareness was evident in both of the studies described here, the nature of these respective disturbances provide somewhat different insights.

In Study 1, the instruction to ignore salient but task-irrelevant somatic cues seems to have precipitated a significant reduction of general attentional efficiency in depressed individuals. Although discussion of the cognitive or neurological mechanisms which may underlie this effect is speculative, a substantial and potentially relevant literature exists which relates similar patterns of reduced attentional efficiency to activity in the default mode network (DMN) of the brain (Kelly et al., 2008; Sonuga-Barke \& Castellanos, 2007; Weissman et al., 2006). The DMN is known to be active during rest and 'mind-wandering' (e.g., Schooler et al., 2011), and deactivated in response to attentional demands, operating in opposition to a task-positive network (TPN) which mediates focused attention (Fox et al., 2005). Attentional lapses, as indexed by lengthy and variable reaction times (Weissman et al., 2006) and errors (Eichele et al., 2008; Li, Yan, Bergquist, \& Sinha, 2007) during a variety of tasks have been associated with reduced DMN deactivation, while intra-individual variability in response times during an attention task has also been shown in healthy participants to relate to the strength of anticorrelations between the DMN and the TPN (Kelly et al., 2008). Such findings suggest that consistent attentional performance may depend upon efficient DMN deactivation, implying that $\mathrm{DMN}$ dysregulation could underlie inconsistent performance such as that observed in depressed participants in Study 1. 
It is well-established that abnormal patterns of intra-and extra-network resting-state functional connectivity can operate with respect to the DMN in depression (Kaiser, Andrews-Hanna, Wager, \& Pizzagalli, 2015; Mulders, van Eijndhoven, Schene, Beckmann, \& Tendolkar, 2015), and functional imaging studies have further demonstrated that depressed groups can be characterized by reduced DMN deactivation during focused attention, certainly when emotionally relevant stimuli are involved (Grimm et al., 2009; Sheline et al., 2009). It is plausible, therefore, that reductions in attentional efficiency in response to somatic cues could reflect reduced DMN deactivation under these conditions in depressed participants. It is possible to speculate further that DMN hyperactivity (perhaps reflecting an increase in stimulus-independent thought or 'mind-wandering') could be a learned response to unwanted somatic or interoceptive sensation in individuals with persistent or recurrent histories of depression, reflecting clinical theories that posit that persistent depression and anxiety are characterized by experiential avoidance of such sensation via cognitive avoidance strategies such as rumination or worry (Borkovec, Alcaine, \& Behar, 2004; Stroebe et al., 2007; Watkins \& Moulds, 2007). Evidence certainly exists (Kam \& Handy, 2013; Kam, Xu, \& Handy, 2014) that mind-wandering can reduce both the perceived intensity of sensory stimuli and their selection by attention, suggesting that this may be an effective, if implicit, strategy for attenuating aversive sensation. If stimulus-independent thought as a means of cognitive avoidance is thus repeatedly negatively reinforced in persistently depressed individuals, may become an habitual response to unwanted somatic sensation, providing a possible explanation for the reduction in attentional efficiency seen in depressed participants in response to somatic cues in Study 1.

An alternative account suggests that high response variability in the depressed group could result from peristimulus efforts to refocus attention centrally in the attempt to ignore cues as instructed. Heightened response variability following somatic cues would then indicate greater difficulty in ignoring such cues and more vigorous resulting efforts to endogenously control the locus of attention. Participants were not asked to provide any information about the cognitive strategies 
that they had used during the task and so further explication of this finding must await additional research.

In Study 2, on the other hand, no significant reductions in overall attentional efficiency were observed, but there was instead a depression-specific exacerbation of validity effects with respect to somatic cues. This suggests that attention within the depressed group was disproportionately captured by somatic cues, and is consistent with the hypothesis that the salience of somatic sensation may be amplified in persistent depression. There is a well-documented and often disabling impact of somatic symptomatology in persistent depression, in addition to painful emotional experience, and increased attentional capture by bodily signals may reflect their heightened salience in this population as a result of their frequently threatening or aversive nature. The proposed increase in the salience, or threat value, of somatic signals in persistent depression is also consistent with the proposal that they may be disproportionately likely to trigger cognitive avoidance strategies.

The studies described here are limited by their modest sample sizes, although a substantial number of observations were collected overall. It is encouraging that the findings were broadly consistent across both studies, but nevertheless replication with a larger sample size would be valuable before drawing definitive conclusions.

While the present studies suggest that paradigms including somatic stimuli can offer a fruitful approach to the investigation of bodily awareness in depression, further work is needed to provide a clearer understanding of the mechanisms involved. In particular, although there are good reasons to believe that somatic and visceral or interoceptive stimuli may share representations at a relevant level of processing, this hypothesis requires direct comparison of interoceptive and exteroceptive somatic paradigms for confirmation. Thus, although the current study may inform concepts of general bodily awareness in depression, it is possible that it speaks only to specifically 
exteroceptive somatic processing. Further research is needed to elucidate the nature of any relationship between somatic and interoceptive awareness and of the underlying brain networks.

Evidence for the disproportionate salience of somatic signals in persistently depressed individuals has potential clinical relevance, particularly with respect to the development of psychological therapies for this population. Somatic symptoms are associated with both depression persistence (Hung, Liu, Wang, Juang, \& Yang, 2010) and treatment resistance (Papakostas et al., 2003) and yet mainstream psychological interventions typically fail to theorise or directly target this aspect of depression phenomenology. The current findings are consistent with the hypothesis that somatic signals are salient in persistent depression (perhaps due to high threat value or expected aversiveness) and as a result are capable of precipitating efforts at cognitive avoidance, particularly when the sensations are explicitly unwanted. This hypothesis parallels and extends existing models of mechanisms of cognitive avoidance such as rumination and worry, which have been theorised as a means of avoiding aversive emotional experience (e.g., Borkovec \& Lyonfields, 1993; Hayes, Wilson, Gifford, Follette, \& Strosahl, 1996; Moore \& Garland, 2004). Further investigation of the subjective meanings associated with salient bodily sensation, its relationships to cognitive and behavioural avoidance and its consequences with respect to depression maintenance would be of assistance in the development of targeted psychological therapies. In addition, the likelihood that attentional processes may be key determinants of altered somatic experience in recurrent depression is of potential clinical interest given that these are in general consciously modifiable through training. For example, Mindfulness-Based Cognitive Therapy (MBCT: Segal, Williams, \& Teasdale, 2002), which entails a substantial emphasis on attention training and includes dedicated body-focused practices, has been shown to offer unique benefits to individuals with more persistent forms of depression (Kuyken et al., 2015; Ma \& Teasdale, 2004; Teasdale et al., 2000; Williams et al., 2014), although the contribution of specifically body-focused attention training is not yet known. In general the current findings may indicate an increased emphasis on body-focused interventions such as relaxation training and body-focused meditation practices with persistently-depressed populations. 
In summary, we demonstrate the utility of exteroceptive somatic paradigms in the study of bodily awareness in depression. Our results indicate that somatic awareness is specifically disrupted in persistent forms of depression and that attentional processes are implicated in this disruption. They also suggest that, at least in some circumstances, the salience of somatic sensation is amplified, rather than attenuated, in persistent depression. Finally, they raise the possibility that in longstanding depression, unwanted somatic sensation may trigger cognitive avoidance strategies with the unintended consequence of disrupted attentional performance on a more general level. These proposals are consistent with existing clinical theory and have relevance for the development of psychotherapeutic approaches to somatic symptomatology in recurrent depression.

\section{Acknowledgements}

We would like to acknowledge the contribution of all participants in both experiments.

\section{References}

Adolfi, F., Couto, B., Richter, F., Decety, J., Lopez, J., Sigman, M., . . Ibanez, A. (2017). Convergence of interoception, emotion, and social cognition: A twofold fMRI meta-analysis and lesion approach. Cortex, 88, 124-142. doi:10.1016/j.cortex.2016.12.019

Ainley, V., Apps, M. A., Fotopoulou, A., \& Tsakiris, M. (2016). 'Bodily precision': a predictive coding account of individual differences in interoceptive accuracy. Philos Trans R Soc Lond B Biol Sci, 371(1708). doi:10.1098/rstb.2016.0003

Association, A. P. (2013). Diagnostic and statistical manual of mental disorders. BMC Med, 17, 133137.

Augustyn, C., \& Peters, M. (1986). On the relation between footedness and handedness. Perceptual and Motor Skills, 63(3), 1115-1118. 
Barrett, L. F., Quigley, K. S., \& Hamilton, P. (2016). An active inference theory of allostasis and interoception in depression. Philos Trans $R$ Soc Lond B Biol Sci, 371(1708). doi:10.1098/rstb.2016.0011

Beck, A. T., Epstein, N., Brown, G., \& Steer, R. A. (1988). An inventory for measuring clinical anxiety: psychometric properties. J Consult Clin Psychol, 56(6), 893-897.

Beck, A. T., Steer, R. A., Ball, R., \& Ranieri, W. (1996). Comparison of Beck Depression Inventories -IA and -II in psychiatric outpatients. J Pers Assess, 67(3), 588-597. doi:10.1207/s15327752jpa6703_13

Bellgrove, M. A., Hester, R., \& Garavan, H. (2004). The functional neuroanatomical correlates of response variability: evidence from a response inhibition task. Neuropsychologia, 42(14), 1910-1916. doi:10.1016/j.neuropsychologia.2004.05.007

Bevan, A. (2020). Somatic salience and sensory precision in persistent depression (Doctoral thesis). https://doi.org/10.17863/CAM.60625

Borkovec, T. D., Alcaine, O., \& Behar, E. (2004). Avoidance theory of worry and generalized anxiety disorder. Generalized anxiety disorder: Advances in research and practice, 2004.

Burton, H., Videen, T. O., \& Raichle, M. E. (1993). Tactile-vibration-activated foci in insular and parietalopercular cortex studied with positron emission tomography: mapping the second somatosensory area in humans. Somatosens Mot Res, 10(3), 297-308.

Bylsma, L. M., Morris, B. H., \& Rottenberg, J. (2008). A meta-analysis of emotional reactivity in major depressive disorder. Clin Psychol Rev, 28(4), 676-691. doi:10.1016/j.cpr.2007.10.001

Cauda, F., D'Agata, F., Sacco, K., Duca, S., Geminiani, G., \& Vercelli, A. (2011). Functional connectivity of the insula in the resting brain. Neuroimage, 55(1), 8-23. doi:10.1016/j.neuroimage.2010.11.049 
Cerliani, L., Thomas, R. M., Jbabdi, S., Siero, J. C., Nanetti, L., Crippa, A., . . Keysers, C. (2012). Probabilistic tractography recovers a rostrocaudal trajectory of connectivity variability in the human insular cortex. Hum Brain Mapp, 33(9), 2005-2034. doi:10.1002/hbm.21338

Chica, A. B., Bartolomeo, P., \& Lupiáñez, J. (2013). Two cognitive and neural systems for endogenous and exogenous spatial attention. Behavioural brain research, 237, 107-123.

Cloutman, L. L., Binney, R. J., Drakesmith, M., Parker, G. J., \& Lambon Ralph, M. A. (2012). The variation of function across the human insula mirrors its patterns of structural connectivity: evidence from in vivo probabilistic tractography. Neuroimage, 59(4), 3514-3521. doi:10.1016/j.neuroimage.2011.11.016

Coghill, R. C., Talbot, J. D., Evans, A. C., Meyer, E., Gjedde, A., Bushnell, M. C., \& Duncan, G. H. (1994). Distributed processing of pain and vibration by the human brain. J Neurosci, 14(7), 4095-4108.

Cohen J. (1988). Statistical Power Analysis for the Behavioral Sciences. New York, NY: Routledge Academic

Corbetta, M., \& Shulman, G. L. (2002). Control of goal-directed and stimulus-driven attention in the brain. Nat Rev Neurosci, 3(3), 201-215.

Craig, A. D. (2009). How do you feel--now? The anterior insula and human awareness. Nat Rev Neurosci, 10(1), 59-70. doi:10.1038/nrn2555

Craig, A. D. (2010). The sentient self. Brain Struct Funct, 214(5-6), 563-577. doi:10.1007/s00429-010$0248-y$

Damasio, A. R. (2006). Descartes' error: Random House.

Dantzer, R., O'Connor, J. C., Freund, G. G., Johnson, R. W., \& Kelley, K. W. (2008). From inflammation to sickness and depression: when the immune system subjugates the brain. Nat Rev Neurosci, 9(1), 46-56. doi:10.1038/nrn2297 
Davis, K. D., Kwan, C. L., Crawley, A. P., \& Mikulis, D. J. (1998). Functional MRI study of thalamic and cortical activations evoked by cutaneous heat, cold, and tactile stimuli. J Neurophysiol, 80(3), 1533-1546.

Deen, B., Pitskel, N. B., \& Pelphrey, K. A. (2011). Three systems of insular functional connectivity identified with cluster analysis. Cereb Cortex, 21(7), 1498-1506. doi:10.1093/cercor/bhq186

DeRubeis, R. J., Siegle, G. J., \& Hollon, S. D. (2008). Cognitive therapy versus medication for depression: treatment outcomes and neural mechanisms. Nat Rev Neurosci, 9(10), 788-796. doi:10.1038/nrn2345

Dijkerman, H. C., \& de Haan, E. H. (2007). Somatosensory processes subserving perception and action. Behav Brain Sci, 30(2), 189-201; discussion 201-139. doi:10.1017/S0140525X07001392

Dimidjian, S., Hollon, S. D., Dobson, K. S., Schmaling, K. B., Kohlenberg, R. J., Addis, M. E., . . Jacobson, N. S. (2006). Randomized trial of behavioral activation, cognitive therapy, and antidepressant medication in the acute treatment of adults with major depression. J Consult Clin Psychol, 74(4), 658-670. doi:10.1037/0022-006X.74.4.658

Dunn, B. D., Dalgleish, T., Ogilvie, A. D., \& Lawrence, A. D. (2007). Heartbeat perception in depression. Behav Res Ther, 45(8), 1921-1930. doi:10.1016/j.brat.2006.09.008

Dunn, B. D., Galton, H. C., Morgan, R., Evans, D., Oliver, C., Meyer, M., .. . Dalgleish, T. (2010). Listening to your heart. How interoception shapes emotion experience and intuitive decision making. Psychol Sci, 21(12), 1835-1844. doi:10.1177/0956797610389191

Eichele, T., Debener, S., Calhoun, V. D., Specht, K., Engel, A. K., Hugdahl, K., . . Ullsperger, M. (2008). Prediction of human errors by maladaptive changes in event-related brain networks. Proc NatI Acad Sci U S A, 105(16), 6173-6178. doi:10.1073/pnas.0708965105 
Eickhoff, S. B., Lotze, M., Wietek, B., Amunts, K., Enck, P., \& Zilles, K. (2006). Segregation of visceral and somatosensory afferents: an fMRI and cytoarchitectonic mapping study. Neuroimage, 31(3), 1004-1014. doi:10.1016/j.neuroimage.2006.01.023

First, M. B., Spitzer, R. L., Gibbon, M., \& Williams, J. B. (2002). Structured clinical interview for DSM-IVTR axis I disorders, research version, patient edition. Retrieved from

Fox, M. D., Snyder, A. Z., Vincent, J. L., Corbetta, M., Van Essen, D. C., \& Raichle, M. E. (2005). The human brain is intrinsically organized into dynamic, anticorrelated functional networks. Proceedings of the National Academy of Sciences, 102(27), 9673-9678.

Francis, S. T., Kelly, E. F., Bowtell, R., Dunseath, W. J., Folger, S. E., \& McGlone, F. (2000). fMRI of the responses to vibratory stimulation of digit tips. Neuroimage, 11(3), 188-202. doi:10.1006/nimg.2000.0541

Furman, D. J., Waugh, C. E., Bhattacharjee, K., Thompson, R. J., \& Gotlib, I. H. (2013). Interoceptive awareness, positive affect, and decision making in major depressive disorder. J Affect Disord, 151(2), 780-785. doi:10.1016/j.jad.2013.06.044

Fustos, J., Gramann, K., Herbert, B. M., \& Pollatos, O. (2013). On the embodiment of emotion regulation: interoceptive awareness facilitates reappraisal. Soc Cogn Affect Neurosci, 8(8), 911-917. doi:10.1093/scan/nss089

Ghaziri, J., Tucholka, A., Girard, G., Houde, J. C., Boucher, O., Gilbert, G., . . Nguyen, D. K. (2015). The Corticocortical Structural Connectivity of the Human Insula. Cereb Cortex. doi:10.1093/cercor/bhv308

Golaszewski, S. M., Siedentopf, C. M., Koppelstaetter, F., Fend, M., Ischebeck, A., Gonzalez-Felipe, V., . . Gerstenbrand, F. (2006). Human brain structures related to plantar vibrotactile stimulation: a functional magnetic resonance imaging study. Neuroimage, 29(3), 923-929. doi:10.1016/j.neuroimage.2005.08.052 
Grimm, S., Boesiger, P., Beck, J., Schuepbach, D., Bermpohl, F., Walter, M., . . Northoff, G. (2009). Altered negative BOLD responses in the default-mode network during emotion processing in depressed subjects. Neuropsychopharmacology, 34(4), 932-943. doi:10.1038/npp.2008.81

Hu, L., Zhang, L., Chen, R., Yu, H., Li, H., \& Mouraux, A. (2015). The primary somatosensory cortex and the insula contribute differently to the processing of transient and sustained nociceptive and non-nociceptive somatosensory inputs. Hum Brain Mapp, 36(11), 4346-4360. doi:10.1002/hbm.22922

Hung, C. I., Liu, C. Y., Wang, S. J., Juang, Y. Y., \& Yang, C. H. (2010). Somatic symptoms: an important index in predicting the outcome of depression at six-month and two-year follow-up points among outpatients with major depressive disorder. J Affect Disord, 125(1-3), 134-140. doi:10.1016/j.jad.2009.12.010

Hunter, E. C., Sierra, M., \& David, A. S. (2004). The epidemiology of depersonalisation and derealisation. A systematic review. Soc Psychiatry Psychiatr Epidemiol, 39(1), 9-18. doi:10.1007/s00127-004-0701-4

Ibanez, A., Gleichgerrcht, E., \& Manes, F. (2010). Clinical effects of insular damage in humans. Brain Structure and Function, 214(5-6), 397-410.

Jakab, A., Molnar, P. P., Bogner, P., Beres, M., \& Berenyi, E. L. (2012). Connectivity-based parcellation reveals interhemispheric differences in the insula. Brain Topogr, 25(3), 264-271. doi:10.1007/s10548-011-0205-y

James, W. (1894). Discussion: The physical basis of emotion. Psychol Rev, 1(5), 516.

Kaiser, R. H., Andrews-Hanna, J. R., Wager, T. D., \& Pizzagalli, D. A. (2015). Large-Scale Network Dysfunction in Major Depressive Disorder: A Meta-analysis of Resting-State Functional Connectivity. JAMA Psychiatry, 72(6), 603-611. doi:10.1001/jamapsychiatry.2015.0071 
Kam, J. W., Dao, E., Stanciulescu, M., Tildesley, H., \& Handy, T. C. (2013). Mind wandering and the adaptive control of attentional resources. J Cogn Neurosci, 25(6), 952-960. doi:10.1162/jocn_a_00375

Kam, J. W., \& Handy, T. C. (2013). The neurocognitive consequences of the wandering mind: a mechanistic account of sensory-motor decoupling. Front Psychol, 4, 725. doi:10.3389/fpsyg.2013.00725

Kam, J. W., Xu, J., \& Handy, T. C. (2014). I don't feel your pain (as much): the desensitizing effect of mind wandering on the perception of others' discomfort. Cogn Affect Behav Neurosci, 14(1), 286-296. doi:10.3758/s13415-013-0197-z

Kelly, A. M., Uddin, L. Q., Biswal, B. B., Castellanos, F. X., \& Milham, M. P. (2008). Competition between functional brain networks mediates behavioral variability. Neuroimage, 39(1), 527-537. doi:10.1016/j.neuroimage.2007.08.008

Khalsa, S. S., Adolphs, R., Cameron, O. G., Critchley, H. D., Davenport, P. W., Feinstein, J. S., ... Mehling, W. E. (2018). Interoception and mental health: a roadmap. Biological Psychiatry: Cognitive Neuroscience and Neuroimaging, 3(6), 501-513.

Kirmayer, L. J., Robbins, J. M., Dworkind, M., \& Yaffe, M. J. (1993). Somatization and the recognition of depression and anxiety in primary care. Am J Psychiatry, 150(5), 734-741. doi:10.1176/ajp.150.5.734

Kleiner, M., Brainard, D., \& Pelli, D. (2007). What's new in Psychtoolbox-3?

Kuyken, W., Hayes, R., Barrett, B., Byng, R., Dalgleish, T., Kessler, D., .. . Byford, S. (2015). Effectiveness and cost-effectiveness of mindfulness-based cognitive therapy compared with maintenance antidepressant treatment in the prevention of depressive relapse or recurrence (PREVENT): a randomised controlled trial. Lancet, 386(9988), 63-73. doi:10.1016/S0140-6736(14)62222-4 
Leweke, F., Leichsenring, F., Kruse, J., \& Hermes, S. (2012). Is alexithymia associated with specific mental disorders? Psychopathology, 45(1), 22-28. doi:10.1159/000325170

Li, C. S., Yan, P., Bergquist, K. L., \& Sinha, R. (2007). Greater activation of the "default" brain regions $\begin{array}{llll}\text { predicts stop } \quad \text { signal } & \text { errors. }\end{array}$ doi:10.1016/j.neuroimage.2007.07.021

Mazzola, L., Faillenot, I., Barral, F. G., Mauguiere, F., \& Peyron, R. (2012). Spatial segregation of somato-sensory and pain activations in the human operculo-insular cortex. Neuroimage, 60(1), 409-418. doi:10.1016/j.neuroimage.2011.12.072

Mulders, P. C., van Eijndhoven, P. F., Schene, A. H., Beckmann, C. F., \& Tendolkar, I. (2015). Restingstate functional connectivity in major depressive disorder: A review. Neurosci Biobehav Rev, 56, 330-344. doi:10.1016/j.neubiorev.2015.07.014

Mussgay, L., Klinkenberg, N., Rüddel, H., \& D'Agata, F. (1999). Heart beat perception in patients with depressive, somatoform and personality disorders. Journal of Psychophysiology, 13, 27-36.

NICE. (2009). Depression in adults: recognition and management: The National Institute for Health and Care Excellence London.

Oldfield, R. C. (1971). The assessment and analysis of handedness: the Edinburgh inventory. Neuropsychologia, 9(1), 97-113.

Ostrowsky, K., Magnin, M., Ryvlin, P., Isnard, J., Guenot, M., \& Mauguiere, F. (2002). Representation of pain and somatic sensation in the human insula: a study of responses to direct electrical cortical stimulation. Cereb Cortex, 12(4), 376-385.

Papakostas, G. I., Petersen, T., Denninger, J., Sonawalla, S. B., Mahal, Y., Alpert, J. E., . . . Fava, M. (2003). Somatic symptoms in treatment-resistant depression. Psychiatry Res, 118(1), 39-45.

Pariante, C. M., \& Lightman, S. L. (2008). The HPA axis in major depression: classical theories and new developments. Trends Neurosci, 31(9), 464-468. doi:10.1016/j.tins.2008.06.006 
Paulus, M. P., \& Stein, M. B. (2010). Interoception in anxiety and depression. Brain Struct Funct, 214(56), 451-463. doi:10.1007/s00429-010-0258-9

Posner, M. I. (1980). Orienting of attention. Q J Exp Psychol, 32(1), 3-25.

Preusser, S., Thiel, S. D., Rook, C., Roggenhofer, E., Kosatschek, A., Draganski, B., . . Pleger, B. (2015). The perception of touch and the ventral somatosensory pathway. Brain, 138(Pt 3), 540-548. doi:10.1093/brain/awu370

Ratcliffe, M. (2014). The phenomenology of depression and the nature of empathy. Med Health Care Philos, 17(2), 269-280. doi:10.1007/s11019-013-9499-8

Sayar, K., Kirmayer, L. J., \& Taillefer, S. S. (2003). Predictors of somatic symptoms in depressive disorder. Gen Hosp Psychiatry, 25(2), 108-114.

Schachter, S., \& Singer, J. E. (1962). Cognitive, social, and physiological determinants of emotional state. Psychol Rev, 69, 379-399.

Schandry, R. (1981). Heart beat perception and emotional experience. Psychophysiology, 18(4), 483488.

Schooler, J. W., Smallwood, J., Christoff, K., Handy, T. C., Reichle, E. D., \& Sayette, M. A. (2011). Metaawareness, perceptual decoupling and the wandering mind. Trends Cogn Sci, 15(7), 319-326.

Segal, Z. V., \& Teasdale, J. (2018). Mindfulness-based cognitive therapy for depression: Guilford Publications.

Seth, A. K. (2013). Interoceptive inference, emotion, and the embodied self. Trends Cogn Sci, 17(11), 565-573. doi:10.1016/j.tics.2013.09.007

Seth, A. K., \& Friston, K. J. (2016). Active interoceptive inference and the emotional brain. Philos Trans R Soc Lond B Biol Sci, 371(1708). doi:10.1098/rstb.2016.0007 
Sheline, Y. I., Barch, D. M., Price, J. L., Rundle, M. M., Vaishnavi, S. N., Snyder, A. Z., . . Raichle, M. E. (2009). The default mode network and self-referential processes in depression. Proc Natl Acad Sci U S A, 106(6), 1942-1947. doi:10.1073/pnas.0812686106

Siedentopf, C. M., Heubach, K., Ischebeck, A., Gallasch, E., Fend, M., Mottaghy, F. M., ... Golaszewski, S. M. (2008). Variability of BOLD response evoked by foot vibrotactile stimulation: influence of vibration amplitude and stimulus waveform. Neuroimage, 41(2), 504-510. doi:10.1016/j.neuroimage.2008.02.049

Simon, G. E., VonKorff, M., Piccinelli, M., Fullerton, C., \& Ormel, J. (1999). An international study of the relation between somatic symptoms and depression. N Engl J Med, 341(18), 1329-1335. doi:10.1056/NEJM199910283411801

Sonuga-Barke, E. J., \& Castellanos, F. X. (2007). Spontaneous attentional fluctuations in impaired states and pathological conditions: a neurobiological hypothesis. Neurosci Biobehav Rev, 31(7), 977986. doi:10.1016/j.neubiorev.2007.02.005

Spence, C. (2010). Crossmodal spatial attention. Annals of the New York Academy of Sciences, 1191(1), 182-200.

Stroebe, M., Boelen, P. A., Van Den Hout, M., Stroebe, W., Salemink, E., \& Van Den Bout, J. (2007). Ruminative coping as avoidance. European archives of psychiatry and clinical neuroscience, 257(8), 462-472.

Terhaar, J., Viola, F. C., Bar, K. J., \& Debener, S. (2012). Heartbeat evoked potentials mirror altered body perception in depressed patients. Clin Neurophysiol, 123(10), 1950-1957. doi:10.1016/j.clinph.2012.02.086

Vaccarino, A. L., Sills, T. L., Evans, K. R., \& Kalali, A. H. (2008). Prevalence and association of somatic symptoms in patients with Major Depressive Disorder. J Affect Disord, 110(3), 270-276. doi:10.1016/j.jad.2008.01.009 
Watkins, E., \& Moulds, M. L. (2007). Reduced concreteness of rumination in depression: A pilot study. Personality and Individual Differences.

Weissman, D. H., Roberts, K. C., Visscher, K. M., \& Woldorff, M. G. (2006). The neural bases of momentary lapses in attention. Nat Neurosci, 9(7), 971-978. doi:10.1038/nn1727

Wiech, K., Jbabdi, S., Lin, C. S., Andersson, J., \& Tracey, I. (2014). Differential structural and resting state connectivity between insular subdivisions and other pain-related brain regions. Pain, 155(10), 2047-2055. doi:10.1016/j.pain.2014.07.009

Wohleb, E. S., Franklin, T., Iwata, M., \& Duman, R. S. (2016). Integrating neuroimmune systems in the neurobiology of depression. Nat Rev Neurosci, 17(8), 497-511. doi:10.1038/nrn.2016.69

zu Eulenburg, P., Baumgartner, U., Treede, R. D., \& Dieterich, M. (2013). Interoceptive and multimodal functions of the operculo-insular cortex: tactile, nociceptive and vestibular representations. Neuroimage, 83, 75-86. doi:10.1016/j.neuroimage.2013.06.057

\section{Supplemental Materials}

\section{Study 1 Medication-Free Sensitivity Analysis}

$n=6$ medicated participants, all belonging to the depressed group, were excluded from the sensitivity analysis. Group effects were re-examined with $N=26$ (10 depressed and 16 healthy) unmedicated participants included in the analysis.

\section{Reaction Time (RT) Data}

The overall main effect of Group was no longer statistically significant, $F(1,24)=2.97, p=$ $0.098, \eta_{p}^{2}=0.11$, but the interaction between Modality and Group persisted, $F(1,24)=5.49, p=$ $0.028, \eta_{p}{ }^{2}=0.19$. As in the main analysis, there was a statistically significant group difference in RT during somatic blocks, $F(1,24)=4.74, p=0.04, \eta_{p}^{2}=0.17$, but not during auditory blocks, $F(1,24)=$ $1.57, p=0.22, \eta_{p}^{2}=0.06$ 
The 3-way interaction between Block, Validity and Group apparent in the main analysis remained statistically significant, $F(1,30)=8.76, p=0.007, \eta_{p}^{2}=0.27$, with a group difference in validity effect evident during later blocks, $\left.F(1,24)=5.25, p=0.031, \eta_{p}{ }^{2}=0.18\right)$, but not during earlier ones, $F(1,24)=0.007, p=0.94, \eta_{p}^{2}<0.001$.

As in the main analysis, no other group effects reached statistical significance.

\section{Error data}

The interaction between Modality and Group no longer reached statistical significance, $F(1$, $24)=3.16, p=0.088, \eta_{p}{ }^{2}=0.12$, and group differences in somatic errors did not survive a correction for unequal variances, corrected $t(10.10)=1.84, p=0.085, d=0.91$. Effect sizes, however, remained medium-large.

As in the main analysis, there was no evidence for any group difference in errors during auditory blocks, $t(24)=0.36, p=0.72, d=0.14)$.

\section{Intra-Individual Variability (IIV)}

The interaction between Modality and Group persisted, $F(1,24)=6.25, p=0.02, \eta_{p}^{2}=0.21$. The group difference in IIV during auditory blocks no longer reached statistical significance, $t(24)=$ $1.76, p=0.092, d=0.69$, but the group difference in IIV during somatic blocks remained significant, $t(24)=3.66, p=0.001, d=1.40$. Within the depressed group, IIV remained significantly greater during somatic than during auditory blocks, $t(9)=-2.37, p=0.042, d=0.51$. 